\title{
肌酸激酶在低浓度胍中失活并非起因 于二聚体的解聚
}

\author{
刘国华侯立向部承鲁 \\ （中国科学院生物物理研究所分子酶学实验室,北京）
}

本实验室的早期工作已经表明, 当肌酸激酶 (ATP-肌酸磷酸转移酶, EC 2, 7,3,2) 用 胍或腿变性时,很低浓度的变性剂就可使酶失活; 但需更高浓度的胍或腿才能引起用一般物化 方法可以观察到的酶的构象变化 ${ }^{[1,2]}$. 此外, 在变性剂浓度相同时, 酶的失活速度比酶分子整 体构象的松散速度快几个数量级 ${ }^{[2,3]}$. 肌酸激酶是二聚体酶, 一般认为稀浓度的变性剂会引起 塞聚酶的解聚。虽然一些作者认为肌酸激酶在单体状态下可能有活 力 力, $^{[4]}$, 但是 Degani 和 Degan ${ }^{[6]}$ 已提出证据, 认为酶必须在二聚体状态下才是有活力的. 因此, 我们的上述实验 结果可以解释为: 酶在低浓度脉或胍中的失活是由于二聚体解聚为单体所致. 较高浓度的变 性剂才引起失去活力的单体的整体构象松散, 而这种构象松散才是可用多种方法监测到的. 本工作的目的是研究酶在足以引起失活但还未引起明显构象变化的低浓洨胍溶液 中的聚合 态. 结果表明, 酶在 $0.5 \mathrm{~mol} / 1$ 胍中活力虽已耂失 $95 \%$,但仍为二聚体.

\section{一、材料与方法}

兔肌肌酸激酶的制备和纯化同文献 [1]. 蛋白浓度测定由 $280 \mathrm{~nm}$ 处的吸收值及吸收系数 $A_{\text {icm }}^{18}=8.8$ 来计算. 盐酸胍为市售分析纯商品, 用前进行两次重结晶. 其它均为分析纯试 剂.

1. 粘度测定 用 Buggell 和 Tanford 方法 ${ }^{[7]}$ 在 $20 \pm 0.01^{\circ} \mathrm{C}$ 下进行. 所用粘度计为 Ostwald 和 Ubbelohde 粘度计两种. Ubbelohde 粘度计用于 $4 \mathrm{~mol} / 1$ 胍变性酶的粘度测定, Ostwald 粘度计则用于其它条件下的粘度测定.

2. 楼胶过滤按 Reiland ${ }^{[83}$ 所述的方法进行, Sephadex G-150, $90 \times 1.6 \mathrm{~cm} \mathrm{LKB}$ 柱. 一组已知分子量的标准蛋白 (Pharmacia 产品) 作为对照. 这组蛋白包括: 醛缩酶 (分子 量为 158000), 牛血清白蛋白 (67000), 卵清蛋白 (43000), 胰凝乳蛋白酶原 $\mathrm{A}(25000)$ 和核糖 核酸酶 $\mathrm{A}(13700)$. 变性酶在 $0.5 \mathrm{~mol} / 1$ 胍中的分子量由 $K_{\mathrm{av}}$ 对 $\log M$ 作图得到, 这里 $M$ 是 实验中蛋白质的分子量, 而且

$$
K_{\mathrm{av}}-\frac{V_{c}-V_{0}}{V_{t}-V_{0}}
$$

$V_{c}, V_{t}$ 和 $V_{0}$ 分别是蛋白质的洗脱体积, 柱床的总体积和蓝色淢聚糖 2000 测得的空水体 积. $V e$ 通过称重得到.

本文 1987 年11月2日收到. 


\section{二、殸 果}

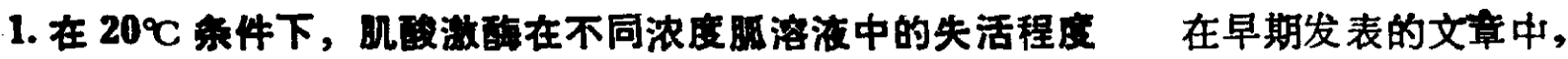
该酶在不同浓度胍溶液中的失活程度都是在 $30^{\circ} \mathrm{C}$ 条件下进行的。但是在这个温度下,特别是: 在延长保温时间情况下,样品溶液变混浊, 干扰粘度测定. 因此酶的失活程度改在 $20^{\circ} \mathrm{C}$ 条件 下进行. 表 1 列举了所得结果, 与 $30^{\circ} \mathrm{C}$ 条件下所得结果差别不大.

2. 相对站度肌酸敫在不同浓度胍溶液中变性失活, 其粘度测定结果如图 1 所示. 由图 1 可见,当胍浓度低于 $0.5 \mathrm{~mol} / 1$ 时, 酶溶液的相对粘度变化不大. 表 1 的结果表明, 0.3 $\mathrm{mol} / 1$ 的畖就已带来明显失活. 当胍浓度为 $0.5 \mathrm{~mol} / 1$ 时，酶失活已经超过 $95 \%$ 。当胍浓度 高于 $1 \mathrm{~mol} / 1$ 时,酶液的相对粘度明显增加. 这大概是由于天然酶分子的松散,或者是由于水 合作用的增加，也有可能是由于两种因素共同作用的结果．我们也曾报道过 ${ }^{[2]}$ ，以光吸收、荻 光、圆二色性以及内埋疏基的暴露作为监测手段,在胍的浓度高于 $1 \mathrm{~mol} / 1$ 时,酶的构象开始发

表 1 肌酸激酶在不同浓度胍溶液中的失活程度

\begin{tabular}{|c|c|c|c|c|c|}
\hline \multirow{2}{*}{$\begin{array}{c}\text { 盐 洃 度 } \\
(\mathrm{mol} / \mathrm{l})\end{array}$} & 剩 & 余 & 活 & ts & $\%$ \\
\hline & $20^{\circ} \mathrm{C}$ & & & & $30^{\circ} \mathrm{C}$ \\
\hline 0 & 100 & & & & 100 \\
\hline 0.3 & 20 & & & & 30 \\
\hline 0.5 & 3.9 & & & & 3.4 \\
\hline 1.0 & 0 & & & & 0 \\
\hline
\end{tabular}

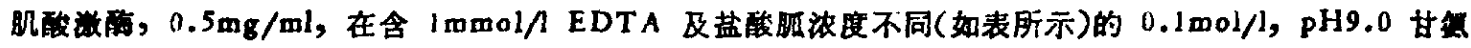

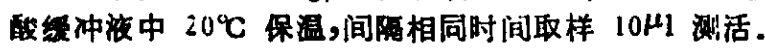

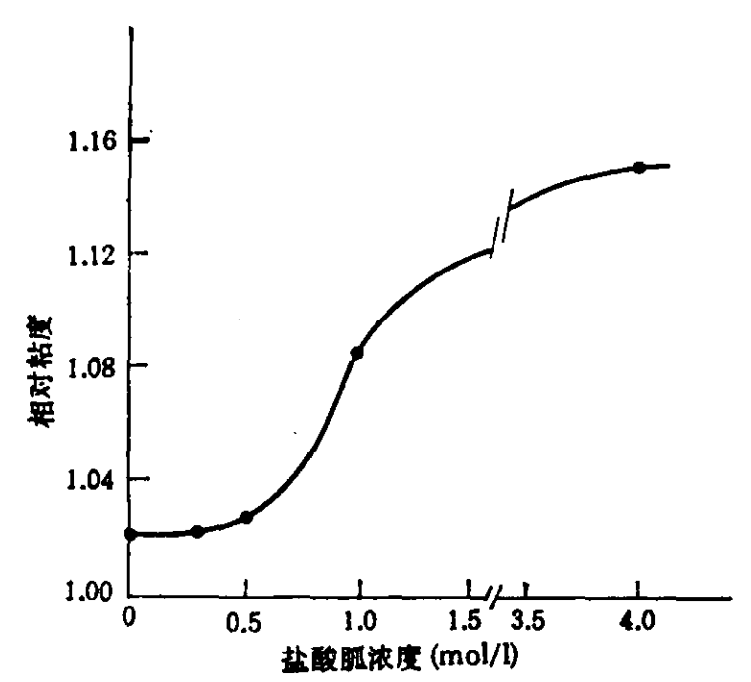

图 1 肌酸放醇在不同浓度胍溶液中的 相对粘度

肌酸敞 $5.3 \mathrm{mg} / \mathrm{ml}$ ，在含 $1 \mathrm{mmol} / \mathrm{EDTA}, 0.1$ $\mathrm{mol} / 1 \mathrm{NaCl}$ 或不同浓度盐酸胍的 $0.1 \mathrm{~mol} / \mathrm{p}, \mathrm{pH}$ 9.0 甘等酸缓冲液中 $20^{\circ} \mathrm{C}$ 保温 $2 \mathrm{~h}$, 再在同样温度下 进行粘度测定

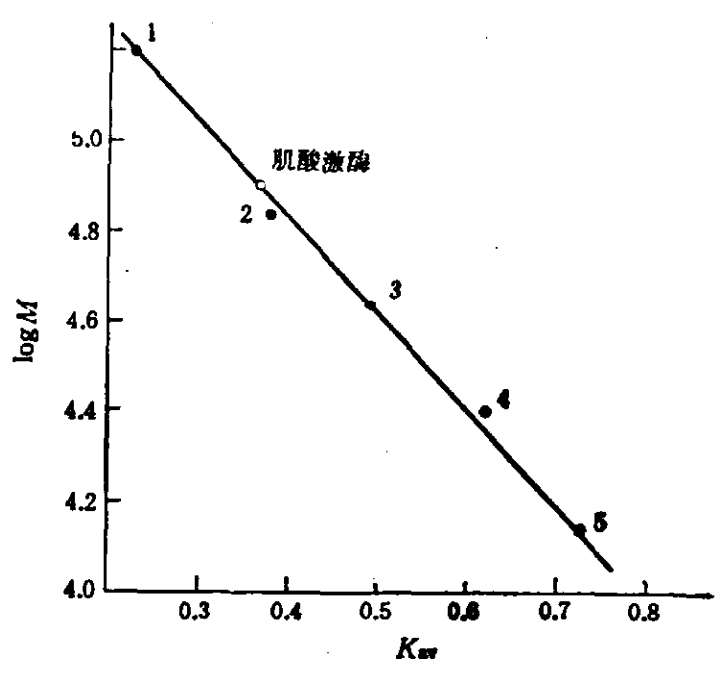

图 2 肌酸激酶与分子量已知的标准蛋白质 资胶过浡比较

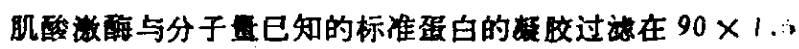

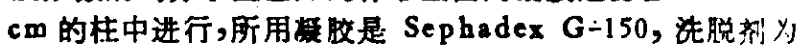
含 $0.2 \mathrm{~mol} / 1 \mathrm{NaCl}$ 和 $1 \mathrm{mmol} / 1 \mathrm{EDTA}$ 的 $0.1 \mathrm{mo} ! / \mathrm{l}$,

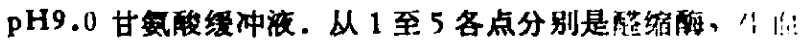

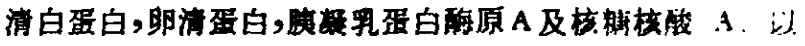

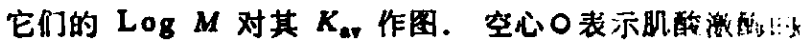
洗脱位罢 
生明显变化. 在 $3 \mathrm{~mol} / 1$ 中用上述方法判断酶分子的松象松散已近于完全. 这也同粘度测定 结果相一致.

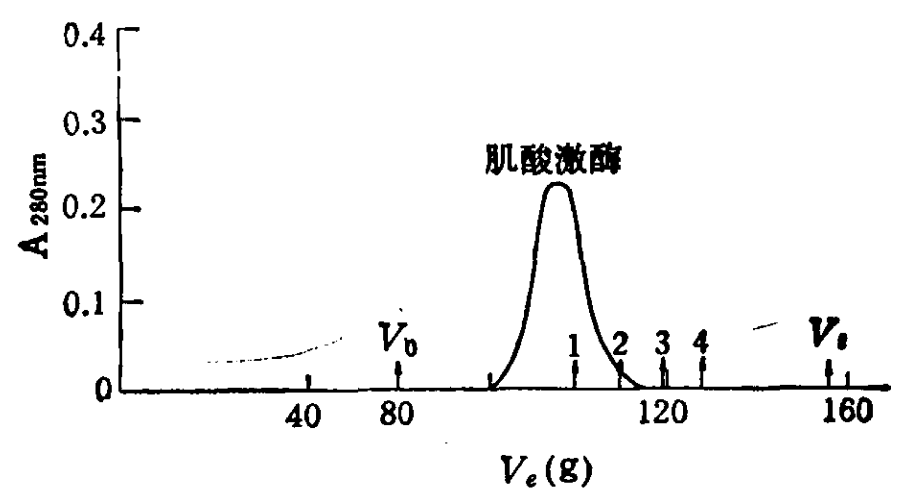

图 3 肌酸激酶在 $0.5 \mathrm{~mol} / 1$ 胍溶液中的凝胶过滤洗脱图 除了酸和其它蛋白上柱前在含 $1 \mathrm{mmol} / 1 \mathrm{EDTA}$ 和 $0.5 \mathrm{~mol} / 1$ 畖的 $0.1 \mathrm{~mol} / 1$ ， $\mathrm{pH} 9.0$ 甘氛酸绥冲液中 $20^{\circ} \mathrm{C}$ 保温 $30 \mathrm{~min}$ 及柱的洗脱也用含相同浓度胍的缕冲

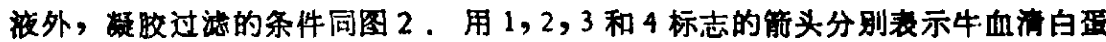

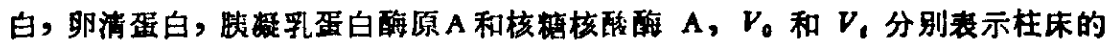
空体积和内体积

3. 㗪胶过滤我们在用 SephadexG-150 比较了一系列已知分子量的蛋白质在无胍及 胍的稀溶液中凝胶过滤行为基础上，进行了肌酸激酶在同样条件下的凝胶过滤实验. 图 2 表 示了它们在无胍溶液中 $\log M$ 对 $K_{\mathrm{av}}$ 的作图关系, 由此得到的肌酸橎酶分子量为 78000 . 这个值与文献用物化方法所测结果一致, 也接近于由它的序列计算所得的二聚体的分子量值 85864. 图 3 表明了肌酸激酶在 $0.5 \mathrm{~mol} / 1$ 畖溶液中凝胶过滤的洗脱图. 由 $\log M$ 对 $K_{\mathrm{av}}$ 作图(图略)所得到的肌酸激酶分子量与图 2 基本相同. 说明这个浓度的胍并不影响酶的分子 量. 图 3 还表明, 肌酸激酶在 $0.5 \mathrm{~mol} / 1$ 胍中的洗脱蜂是单一对称的, 进一步说明在这个畖浓 度下酶的二聚体并没有产生可察觉的解聚。

\section{三、讨 论}

对于 Stoke's 半径不同的球蛋白而言，胶过滤已广泛用于其分子量的测定. 若是非球 状蛋白,亦可用粘度进行校正 ${ }^{[9]}$. 甚至在 $6 \mathrm{~mol} / 1$ 胍中,蛋白质构象完全松散时, 仍然可以得到 满意的结果. 为了确证低浓度胍中肌酸激酶分子构象是否发生变化而影响到凝胶过滤测定分 子量的准确性,本文测定了肌酸激酶在不同浓度胍中的相对粘度.

我们知道,蛋白质的特性粘度决定于该蛋白质分子的形状和水合程度,而用于测定其形状 和水合程度的变化,粘度是几种最灵敏的方法之一，我们工作中通过粘度测定表明,肌酸票酶 在 $0.5 \mathrm{~mol} / 1$ 胍中分子形状并没有发生什么变化. 通过肌酸激酶以及一些常用标准蛋白在稀 的胍溶液中凝胶过滤的洗脱体积测定则表明，它们之中的任一蛋白分子量都没有显示出不规 则的变化. 显然,肌酸激酶的活力在 $0.5 \mathrm{~mol} / 1$ 鲘中央失 $95 \%$ 的同时, 并没有发生二聚体的解 聚。

Da Costa 等人报道了肌酸激酶在不同浓度的腿溶液中粘度的变化 ${ }^{[10]}$. 与 Grossman 的 报道 ${ }^{[4]}$ 相反,胍的浓叜比腺低得多情况下就能引起同样变化, 说明胍对肌酸酶的变性作用比 腿更为有效。值得注意的是, 肌酸激酶在直到 $2 \mathrm{~mol} / 1$ 腿溶液中的粘度都末发生明显变化 ${ }^{[203}$, 
然而这个胍浓度下酶已失活超过 $75 \%$ 。 因此, 在胍与豚两种变性剂条件下,肌酸激酶的失江 都不是由于分子大小及形状变化,而很可能是由于其活性部位的细微构象变化所引起的 ${ }^{2,3]}$ 实 际上,根据引入活性部位的荻光探剂检测结果, Grossman ${ }^{[11]}$ 报道腿变性过程中, 活性中心综 构在变性初期迅速变化.

\section{今 文献}

[ 1 ] Yao, Q.-Z., Hou, L. X., Zhou, H. M. and Tsou, C. L., Sci. Sin, 25B(1982), 1186-1193.

[ 2 ] Yao, Q. Z., Zhou, H. M., Hou, L. X. and Tsou, G. L., Sci. Sin, 25B(1982), 1296-1302.

[ 3 ] Yao, Q. Z., Tian, M. and Tsou, G. L., Sci. Sin, 28B(1985), 484-493.

[4] Grossman, S. H.. Pyle, J. and Steiner, R. J., Biochemistry, 20(1981), 6122-6128.

[5] Bickerstaff, G. F. and Price, N. C., Intern. J. Biochem, 19(1978), 108.

[6] Degani, C. and Degani, Y., J. Biol. Chem, 255(1980), 8221-8228.

[7] Buggel, J. G. and Tanford, G., J. Phys. Chem., 60(1960), 1204.

[8] Reiland, J., Methods Enzymol., 22(1971), 287-321.

[ 9$]$ Grubisic, Z., Rempp, P. and Benoit, H., J. Polymer Sci. Polym. Lett. Ed., 5(1967), 753-759.

「10] Da Costa, W. A. and Friedberg, F., J. Biol. Chem, 235(1960), 3134.

[11] Grossman, S. H., Biochim. Biophys. Acıa, 785(1984), 61-67. 